NOTICE WARNING CONCERNING COPYRIGHT RESTRICTIONS:

The copyright law of the United States (title 17, U.S. Code) governs the making of photocopies or other reproductions of copyrighted material. Any copying of this document without permission of its author may be prohibited by law. 


\title{
A Formal Specification of a Visual Language Editor
}

\author{
Jeannette M. Wing and Amy Moormann Zaremski \\ February 25, 1991 \\ CMU-CS-91-1123
}

\author{
School of Computer Science \\ Carnegie Mellon University \\ Pittsburgh, PA 15213
}

Submitted to Sixth International Workshop on Software Specification and Design.

\begin{abstract}
This paper presents a non-trivial case study on the use of the Larch [GHW85, GHM90] specification languages to describe the Miro visual languages and graphical editor [HMT ${ }^{+}$90]. In addition to excerpts from the specification, we discuss properties of Miró provable from the specification, limitations of Larch, and general lessons learned from this exercise. The companion technical report, CMU-CS-91-111, contains the entire specification.
\end{abstract}

This research was sponsored by the Avionics Lab, Wright Research and Development Center, Aeronautical Systems Division (AFSC), U. S. Air Force, Wright-Patterson AFB, OH 45433-6543 under Contract F33615-90-C-1465, Arpa Order No. 7597. A. Moormann Zaremski is also supported by a fellowship from the Office of Naval Research.

The views and conclusions contained in this document are those of the authors and should not be interpreted as representing the official policies, either expressed or implied, of the U.S. Government. 
Keywords: formal specification, visual language, Larch, Miro 


\section{Introduction to and Contributions of This Paper}

The Miro visual languages let users specify formally through pictures the security configuration of file systems (i.e., which users have access to which files) and general security policy constraints (i.e., rules to which a configuration must conform). With the Miro editor, users draw both types of pictures and access other Miro tools.

This paper presents a non-trivial case study on the use of the Larch specification languages to describe the Miro languages and editor. We had two goals in mind while writing the specification: to end up with a formal specification that could serve as both documentation of and a basis for formal reasoning about the specificand, i.e., the Miró languages and editor; and to apply Larch to determine its strengths and weaknesses. Though there are a growing number of cases studies of formal specifications for formal methods like VDM and $Z$, very few examples of Larch specifications have been published. Moreover, whereas previous Larch papers have emphasized and presented Larch traits, ours presents a balance of both traits and interfaces.

We begin with brief descriptions of Miró and Larch in Section 2 and present excerpts from the specification in Section 3. In Section 4 we discuss properties of the specificand provable from the specification, limitations of Larch, and general lessons learned from this exercise. In Section 5, we close with a brief discussion of related and future work. All of the traits have been checked for syntactic and static semantic correctness using the Larch Shared Language (LSL) Checker and the interface specification has been checked for syntactic and type correctness using the Generic Interface Language (GIL) Checker. ${ }^{1}$ Including blank lines and comments in the code and specification, the code size of the editor is 8400 lines of Common Lisp and the Larch specification is 1035 lines of Larch traits and interfaces.

\section{Miró and Larch}

\subsection{Specificand: Miró}

Miro consists of two visual languages, the instance language and the constraint language [HMT+90]. An instance (language) picture graphically denotes an access matrix that defines which users have which accesses to which files. Instance pictures model the specific security configuration between a set of users and a set of files, e.g., Alice cannot read Bob's mail file. A constraint (language) picture denotes a set of instance pictures (or equivalently, the corresponding set of access matrices) that satisfies a particular security constraint, e.g., users with write access to a file must also have read access. When an instance picture, $I P$, is in the set denoted by a constraint picture, $C P$, we say $I P$ "matches" $C P$
or $I P$ is "legal with respect to" $C P$.

The basic elements in the instance language are boxes and arrows. Boxes that contain no other boxes represent users and files. Boxes can contain other boxes to indicate groups of users and directories of files. User group boxes may overlap to indicate a user is in more than one group. Labeled arrows go from user (group) boxes to file (group) boxes; the label indicates the access mode, e.g., read or write. Access rights are inherited by corresponding pairs of boxes contained within boxes connected by arrows, thus minimizing the number of arrows necessary to draw. Arrows may be negated to indicate the denial of the labeled access.

Figure 1 shows an instance picture, as drawn in the Miro editor. The positive arrow from Alice to Alice's files indicates that Alice has read and write access to her files. The positive arrow from Alice's friends to Alice's schedule file indicates that both Bob and Charlie have read access to Alice's schedule. By default, since there are no arrows between Alice's friends and her other files, Bob and Charlie do not have read access to Alice's mail file. We could also show this property with an explicit negative arrow between Alice's friends and her mail file.

The constraint language also consists of boxes and arrows, but here the objects have different meanings. A box labeled with an expression defines a set of instance boxes. E.g., the left-hand box in Figure 2 denotes the set of instance boxes of type User. There are three types of arrows, allowing us to describe three different relations between boxes

\footnotetext{
${ }^{1}$ The full specification is available as a technical report upon request from the second author. Tools are available upon request from the first
thor. 


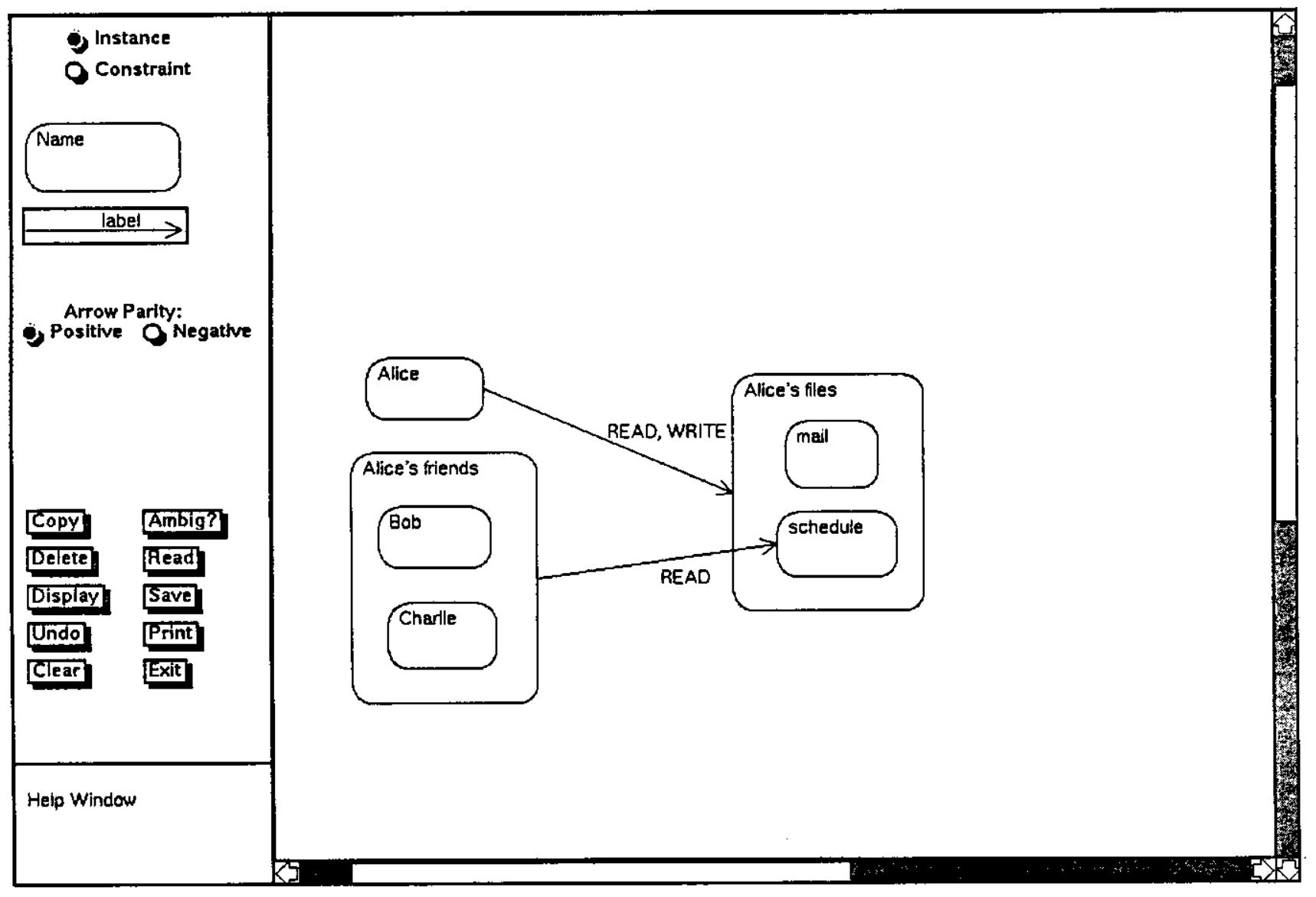

Figure 1: The Miro editor and a sample instance picture

in an instance picture, IP: syntactic (solid horizontal) - whether an arrow explicitly appears in $I P$; semantic (dashed horizontal) - whether an access right exists in the matrix denoted by $I P$; and containment (solid vertical with head inside box) - whether a box is nested within another in IP. Additionally, the thickness attribute of each constraint object is key in defining a constraint picture's meaning: in general, for each set of instance objects that matches the thick part of the constraint, there must be another set of objects (disjoint from the set matching the thick part) that matches the thin part. Figure 2 shows a constraint picture that specifies that users who have write access to a file must have read access to it as well.

The Miro editor provides the facilities to create, view, and modify both instance and constraint pictures. Pictures can be saved in files and read back into the editor. The editor also serves as an interface to other Miro tools, e.g., one that generates the access matrix denoted by an instance picture, and one that translates a picture into PostScript form. The left-hand side of the window in Figure 1 displays a menu from which users can select the type of picture and object they wish to draw. Other menu buttons provide additional editing commands and interfaces to the other Miró tools. The editor maintains language-specific constraints as users draw pictures. For example, all arrows in Miró pictures must be attached to boxes: if a user moves a box in the picture, all arrows attached to that box also move. Our formal specification captures this behavior precisely.

\subsection{Specification Language: Larch}

We wrote our specifications using Larch specification languages. We assume some rudimentary knowledge of Larch, present a refresher here, and give further details as we present the specification. See [GHW85, GHM90] for more 


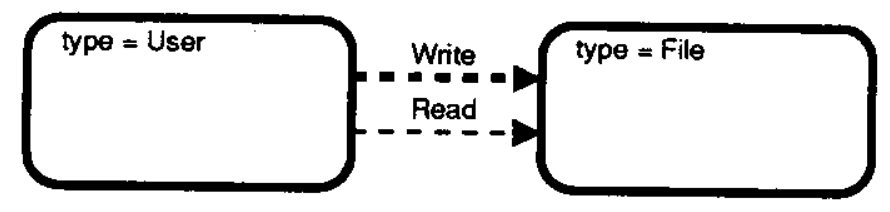

Figure 2: A sample constraint picture

details.

Larch provides a "two-tiered" approach to specification. In one tier, the specifier writes traits in the Larch Shared Language (LSL) to assert state-independent properties of a program. Each trait introduces sorts and operators and defines equality between terms composed of the operators (and variables of the appropriate sorts). E.g., the Box trait (Figure 4) introduces the sort $B$ and the operators move box, resi ze_box, and copy box; four equations constrain the meaning of copy box.

In the second tier, the specifier writes module interfaces in a Larch interface language, such as the Generic Interface Language (GL) [Che89], to describe state-dependent effects of a program. A requires clause states each procedure's pre-condition; an ensures clause, its post-condition; a modifies clause lists those objects whose value may possibly change. The assertion language for the pre- and post-conditions is drawn from LSL traits. Through based on clauses, a Larch interface links to LSL traits by specifying a correspondence between (programming-language specific) types and LSL sorts. An object has a type and a value that ranges over terms of the corresponding sort.

Part of the interface specification for the editor below defines the type Editor, which is based on the Ed sort, introduced in the EditorTrait trait. The MoveBoxes procedure's pre-condition requires that some non-empty set of objects be selected. The post-condition says that the state of the picture in the editor is updated (as defined by the move boxes operator whose meaning is obtained from EditorTrait) and that all objects are unselected. In a postcondition an undecorated formal, $e$, stands for the initial value of the object; a subscripted one, e ! post, stands for the final value. The modifies clause states that MoveBoxes may change only the editor and no other object. ${ }^{2}$

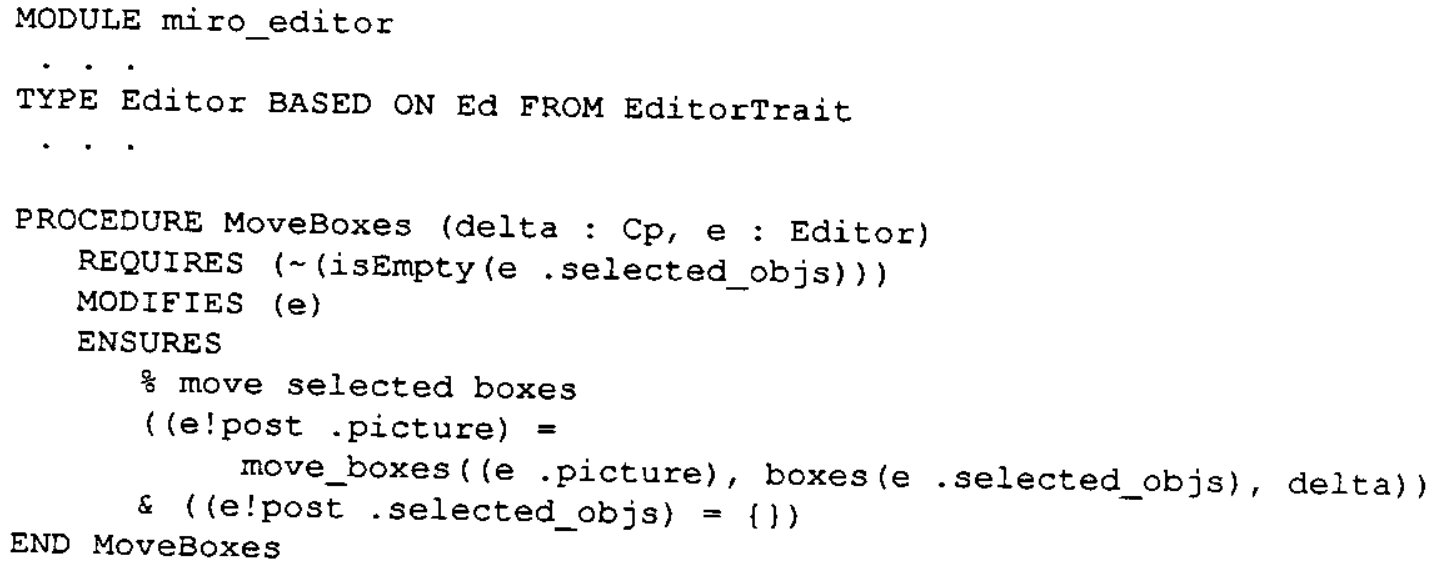

\footnotetext{
${ }^{2}$ Boldfaced keywords in text appear as all capitals in the actual specification. All specifications in this paper appear exactly as they have been checked by the LSL and GILC tools. Thus, non-ascii symbols appear as IAT $\mathrm{E}^{X}$ macros (e.g., $\mid U$ for $U$ (set union)).
} 


\section{The Specification}

We divide the specification into two main pieces: that specifying properties of Miro pictures and that specifying the behavior of the editor. We use LSL to describe Miro pictures and additionally use GIL to specify the editor's procedures that manipulate the pictures.

Figure 3 illustrates how the traits of the LSL part of the specification fit together. Each oval corresponds to a trait and an arrow indicates that one trait includes another. A picture in either the instance or constraint language is a collection of boxes and arrows (BasicPic trait). Miró pictures are further constrained to satisfy well-formedness properties (Pic and WFPic), which include, for example, the condition that arrows be attached to boxes. Pictures drawn in the instance and constraint languages are structurally very similar, so our approach is to factor out the properties common to both languages (bold ovals in Figure 3), and then specialize for each language (dashed ovals). At the bottom we define the EditorTrait which includes all the others; it is the link between the LSL and GIL tiers in the editor specification. In this paper we will walk through only the traits along the spine in the figure. Also, to save space we will typically present only the signature and not the equations in each trait.

\subsection{The Miró Languages}

\subsubsection{Basic Objects}

Boxes and arrows are the basic objects of any Miro picture. Instance and constraint pictures differ only in the attributes of their respective boxes and arrows and in the rules for combining them into pictures. Traits for boxes and arrows are later specialized to distinguish between instance and constraint pictures.

A box object has a value of sort B (see Box trait in Figure 4) and has pos and size attributes. ${ }^{3}$ We assume a box's position is the coordinates of its bottom left corner, and its size is given as a coordinate pair of its width and height. A box also has a label attribute, which will be customized for instance and constraint boxes, and the boolean attributes thick and starred, needed for constraint pictues. Finally, we use sysname to identify a box. Although we do not require it at this level, we assume that sysnames are unique. Sysnames serve two purposes: to distinguish between otherwise identical objects and to provide an easy way to identify objects in a picture for other operations (e.g., deleting a box).

The Box trait also introduces operators on boxes. The record notation in Larch automatically produces the generator for the record sort: an operator that takes as its arguments all of the attributes of the sort and produces something of the record sort (e.g., mk_B). The record shorthand also generates operators of the form b. foo and foo_gets $\left(b, f o o_{-} v a l\right)$ for each field foo (where $b$ is of sort B and foo_val has the same sort as the field foo).

The introduces clause declares additional operators and the equations in the asserts clause give them their meaning. Move_box and resize_box reset the position and size attributes of their respective box arguments. The reason we even need a copy box operator as opposed to relying on Larch's built-in equality operator for all sorts is that not all values of all fields are the same when one box is a copy of the other. Having an explicit copy box operator also makes it convenient to specify default initialization values for certain fields. For example, one issue in the design of the editor was whether or not a copy of a box should have the same label, or should have a default label (the empty string). Thus, we choose instead to write equations only for the fields we require to be the same, and allow the values of the other fields to be specified in another trait or at the interface level.

Boxes in the instance language differ from those in the constraint language in two ways. First, the sort of values for some attributes are different. Namely, an instance box's label is a string whereas a constraint box's label is "box descriptor" - a boolean expression that describes a set of boxes. We handle this difference by using the generic sort LabelSort and then in the InstanceBox trait, we rename LabelSort with the sort Str (for strings) and in the ConstraintBox trait, we rename it with the sort BoxDesc (for box descriptors).

\footnotetext{
${ }^{3}$ The $C P$ son is defined in the CoordPair trait as a record of two integers
} 


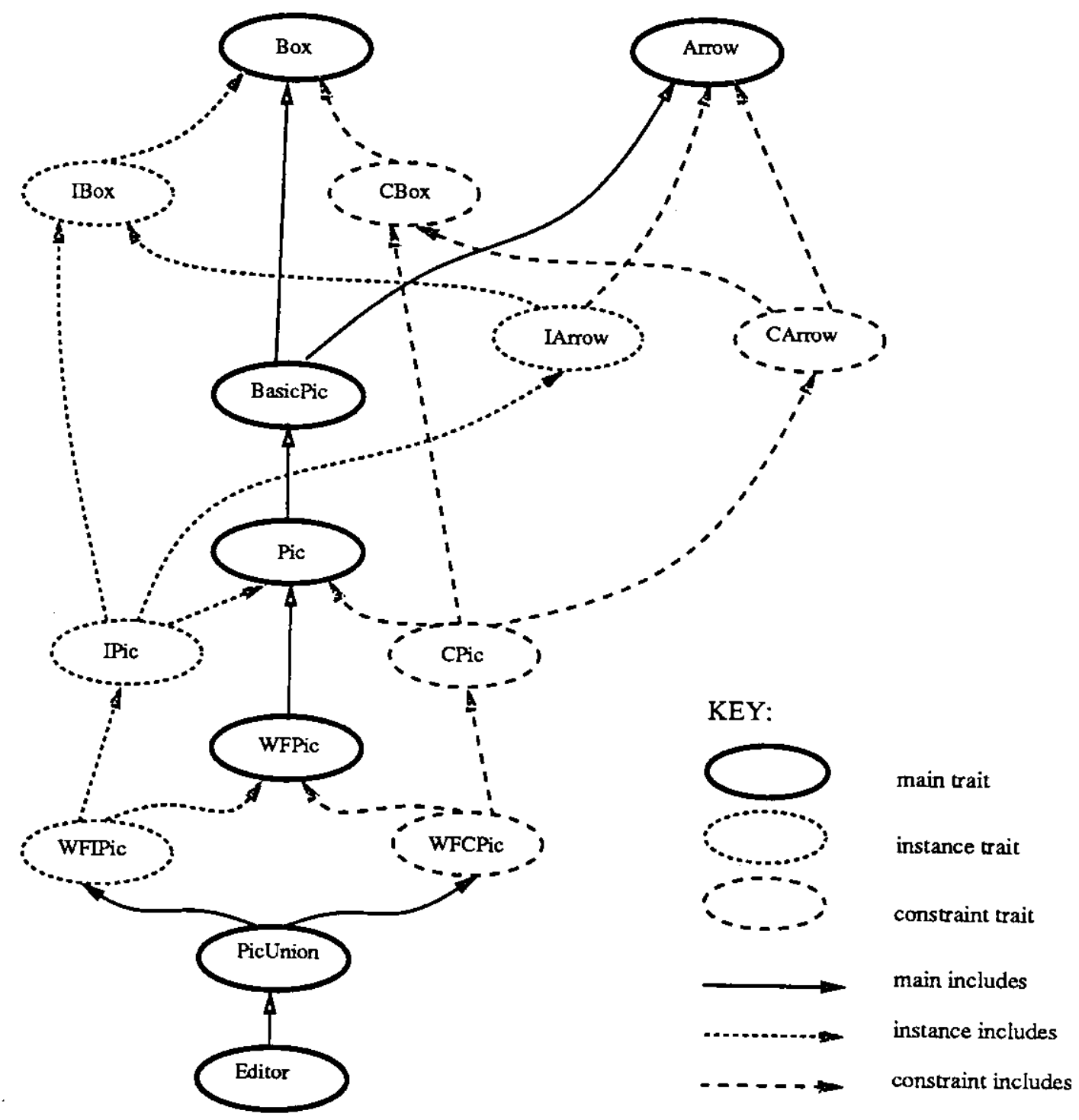

Figure 3: The dependencies of the Miro traits

The second difference is that some attributes are meaningful for only constraint pictures and hence are unnecessary for instance pictures. We could avoid this problem if either Larch provided a way to extend (subtype) records or we were willing to use nested records (see Section 4). However, since there are only a few of these attributes, we choose instead to include at the trait level all possible attributes for the two kinds of boxes, and then make assertions at the interface level that place constraints that will distinguish between instance and constraint boxes.

We specify properties on arrows similarly (using Larch's record construct) and omit the details here. To box and from_box are two of many fields in the record for arrows and are used to keep track of the boxes at the arrow's head and tail.

\subsubsection{Pictures}

A picture is a set of boxes and set of arrows (see Figures $5-9$ ). To avoid a long and confusing trait, we divide the specification of properties of pictures into three main traits: BasicPicture, Picture, and WFPicture 


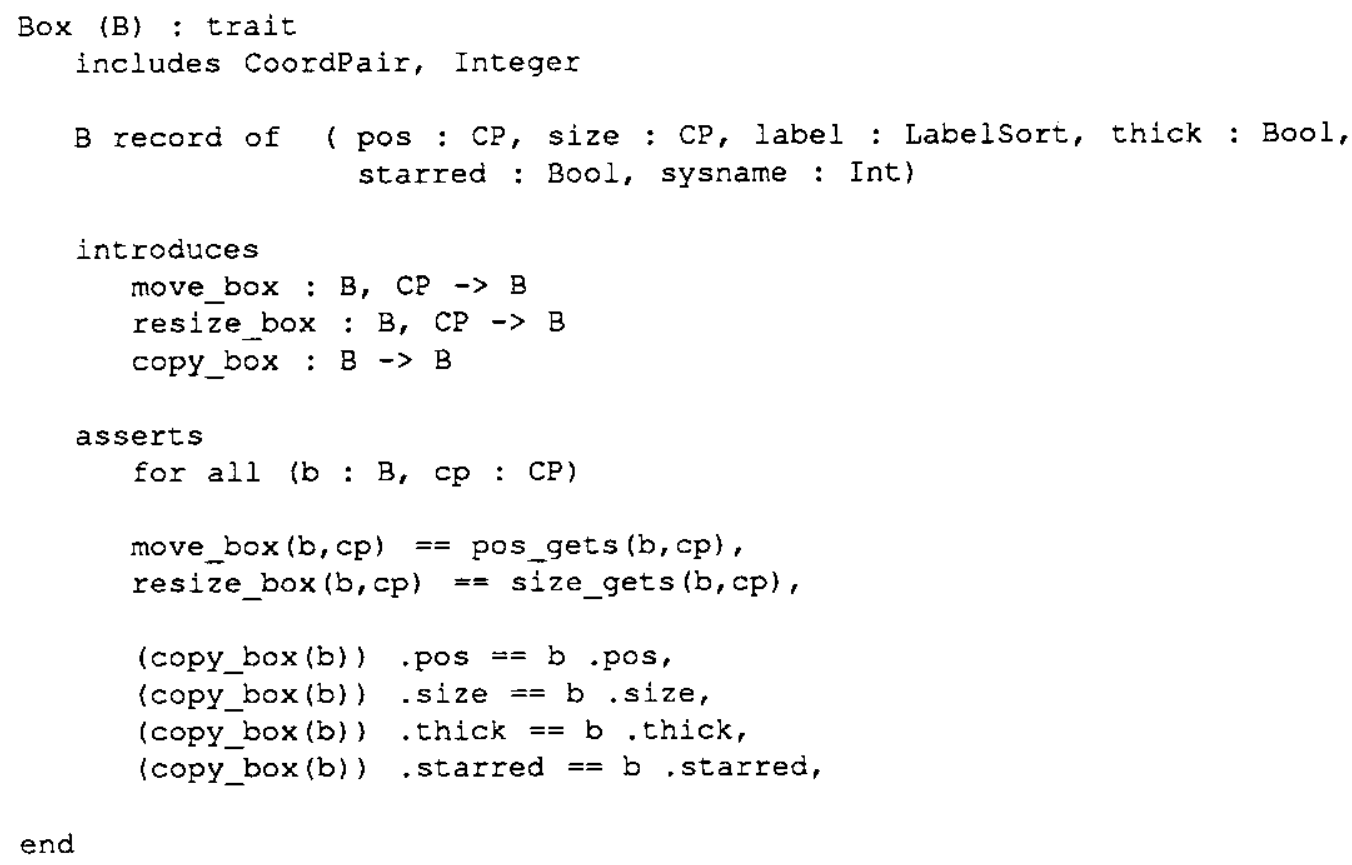

end

Figure 4: The Box Trait

(Well-Formed Picture). BasicPicture introduces the picture sort, Pic, and basic operators on a picture. Picture introduces the sort Object, operators that manipulate objects in the picture, and sorts and operators for the "Change Attribute" interface. Finally, WFP i ct ure introduces the definition of "well-formedness" and operators that build on top of the basic operators to create and manipulate "well-formed" pictures.

The BasicPicture trait (Figure 5) introduces the sort Pic for pictures. The includes clause lets us use all sort and operator names from the included traits with appropriate renamings. E.g., the renaming of sort identifiers in the Set trait (from the Larch Handbook) gives us a sort BSet for sets of boxes and an operator empty BSet to denote the empty set of boxes. The operators that generate a picture are create_picture, insert_box, and insert_arrow. This trait also introduces the operators move_a_box, resize_a_box, delete_box, and delete_arrow. We use pic_union later in traits to perform the higher-level copy operation. boxes and arrows are observer functions on pictures.

The axioms defining these operators are straightforward and given in the standard style of "algebraic" specifications (define the meaning of each non-constructor operator in terms of each constructor operator). We give details of only one here. Copy-picture recurses through the objects in the picture and calls the appropriate copy operator on each object (box or arrow).

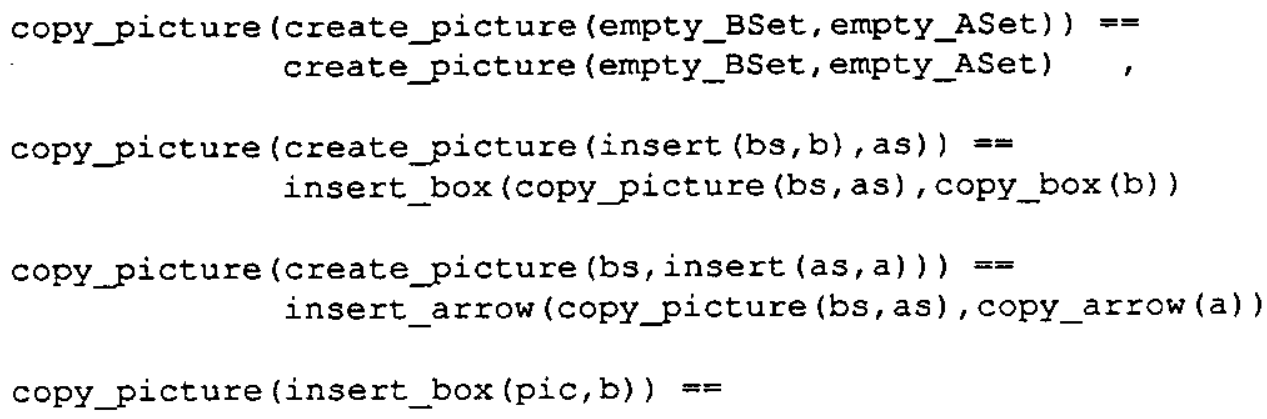




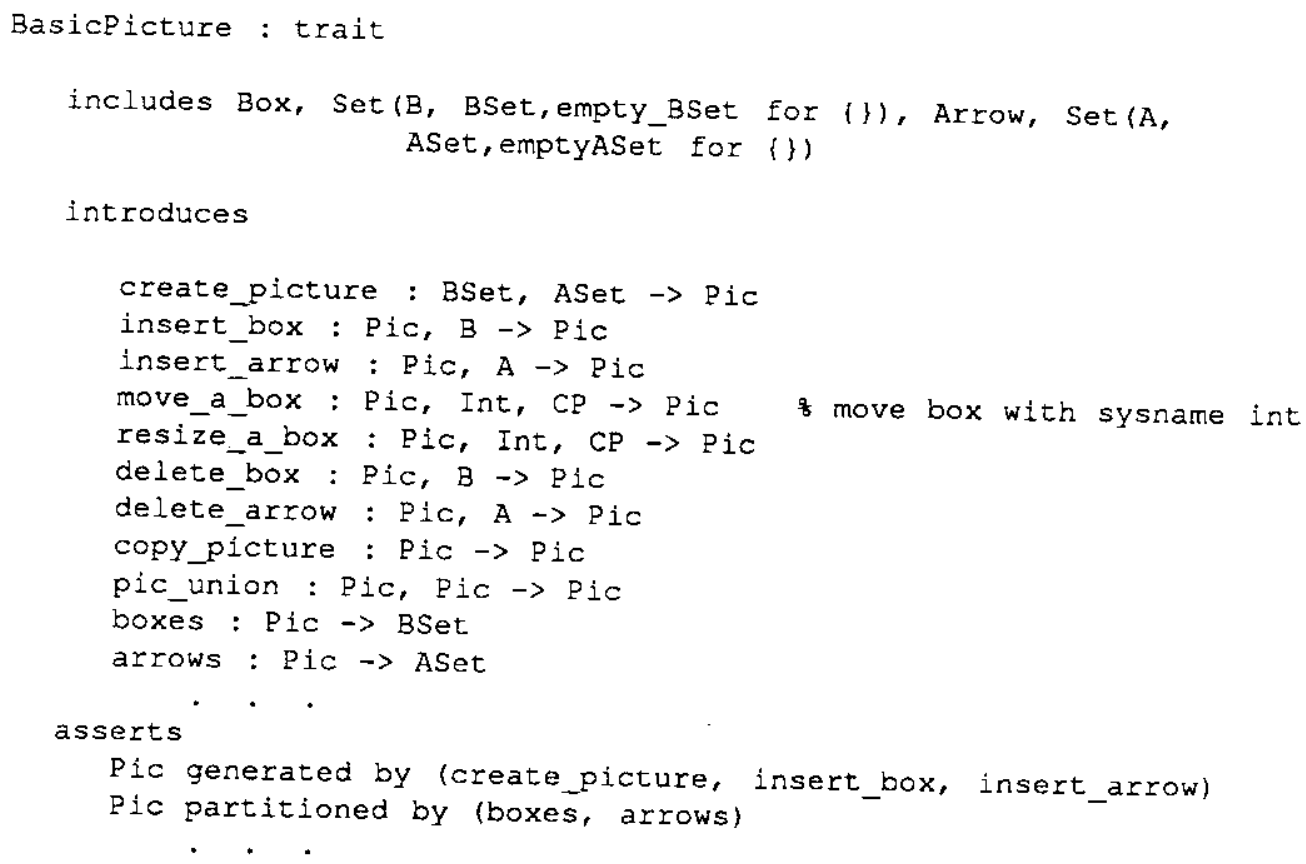

Figure 5: Part of the BasicPicture Trait

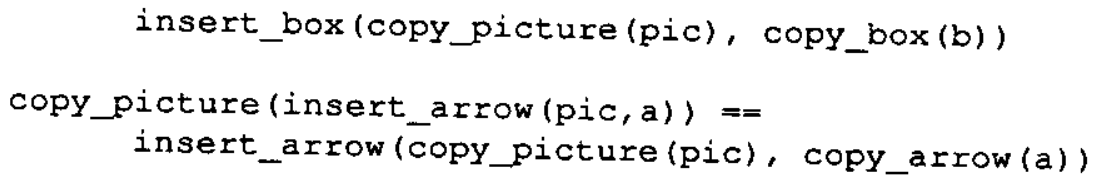

The Picture trait (Figure 6) builds on the BasicPicture trait by introducing a new sort Object. which is a union of the box and arrow sorts. Most of the editor's procedures work regardless of whether its argument is a box or arrow; thus, for each operator common to the box and arrow sorts, we introduce an equivalent operator for the Object
sort.

The operator objects returns the set of all objects in a picture; boxes and arrows extract the sets of boxes and arrows from a set of objects. The operator toggle_in adds the specified object to a set of objects if it is not already in it, otherwise it deletes the object. The editor trait uses toggle in to maintain a set of selected objects in a picture. The operator copy_object is the object analog of the corresponding box and arrow operators. The operator change_att $r$ lets us set the value of an object's attribute and rely on the helping operators valid_att $r$ and valid_value which define which attributes and values are valid for each object. Some of the complexity of this trait results from our decision to use the union construct for objects. The advantage of putting this complexity here is that we keep the interfaces clean and simple; we discuss some of the disadvantages in Section 4.

\subsubsection{Well-formed Pictures}

BasicPicture and Picture traits introduce the picture sort Pic and basic operators on pictures. However, Miro pictures drawn in the editor cannot be an arbitrary collection of boxes and arrows; we require that they be well-formed.

Among the many operators (not all are shown) in the WFPicture trait (Figure 7), we introduce operators for defining well-formedness and extracting a maximal well-formed subset of a set of objects. We only introduce 


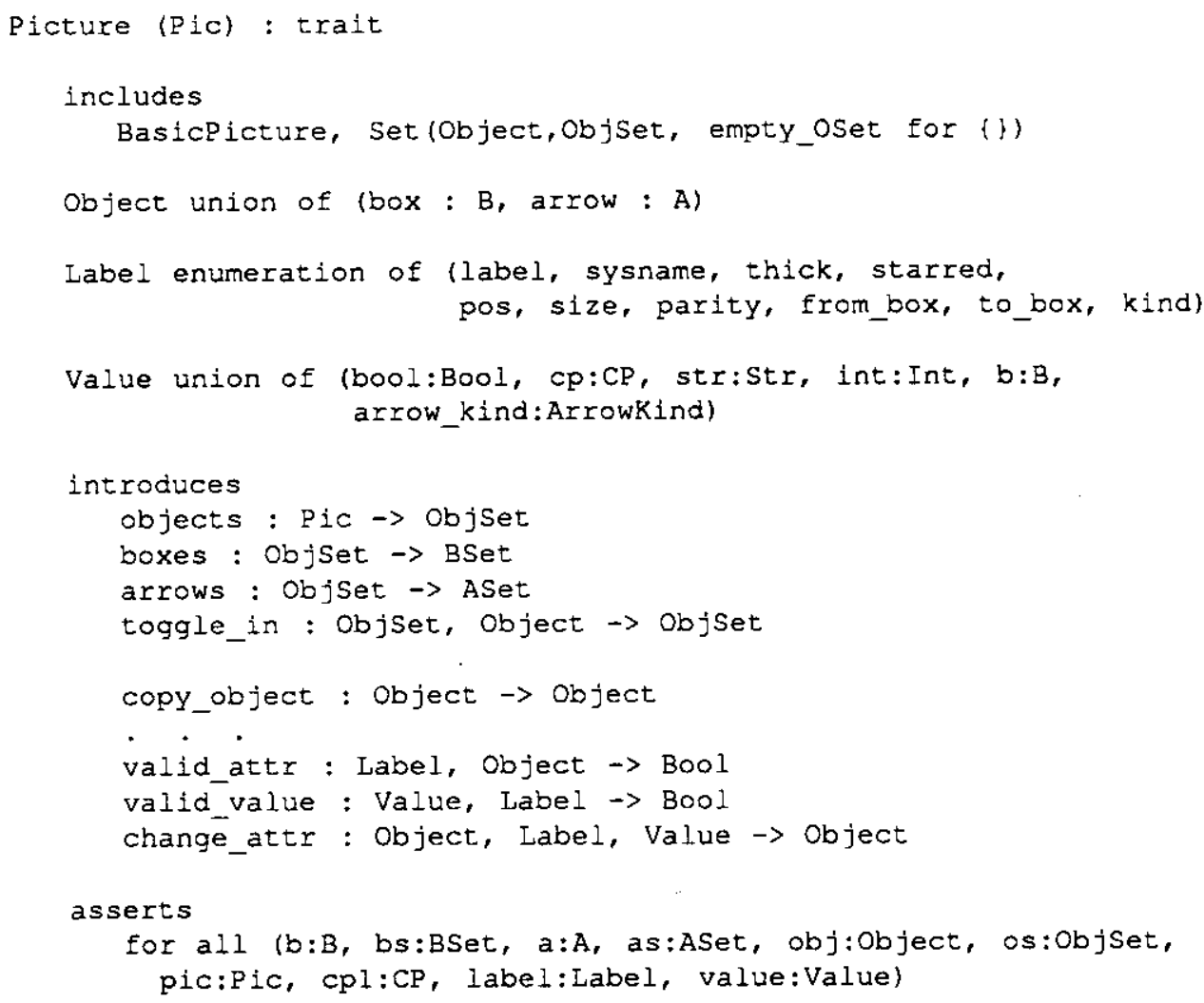

Figure 6: Part of the Picture Trait

but give no equations defining well formed operator in this trait because instance and constraint pictures have different notions of well-formedness. However, since one well-formedness condition common to both instance and constraint pictures is that all arrows must be attached to boxes, we additionally introduce and define here the operator all_arrows_attached. There are other well-formedness conditions, e.g., arrows must go from user boxes to file boxes in an instance picture, but for this paper, we assume only the arrows-attached property.

We use extract.wf in the editor interface to describe the behavior of the command to copy a set of selected objects, which itself is a picture that may or may not be well-formed. The result of ext ract_wf(os) is a picture that contains all the objects of os except the "dangling" arrows (i.e., arrows which are not attached to boxes in os).

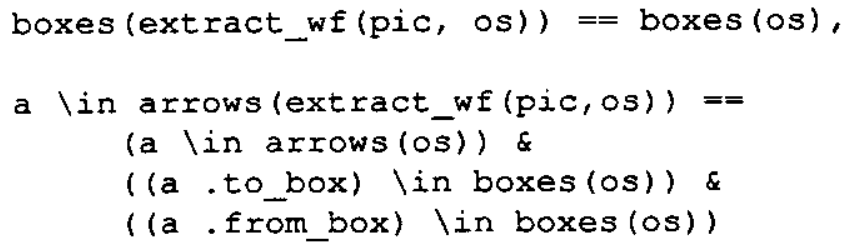

To show how we define properties of a well-formed instance picture, let us look at the WFInstancePicture trait (Figure 8), which includes and specializes the WFPicture trait. The asserts equation states that an instance picture is well_formed if and only if all arrows in the picture are attached. In WFInstancePicture we also introduce the 


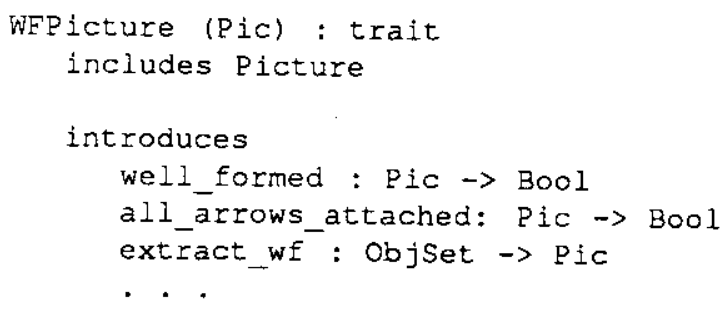

Figure 7: Part of the WellFormedPicture Trait

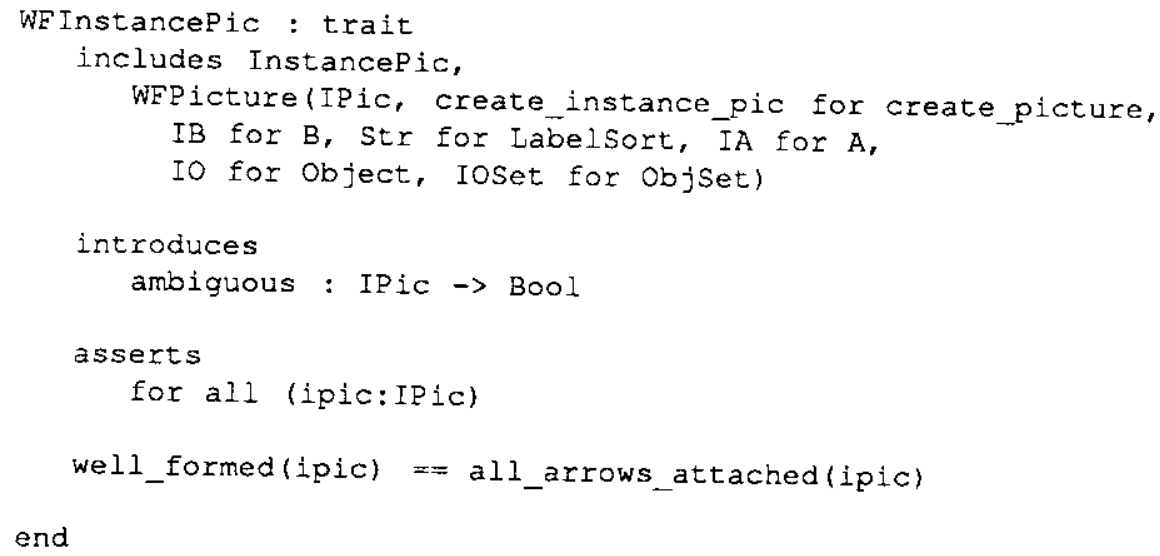

Figure 8: The WellFormed Instance Picture Trait

function ambiguous. Because there are both positive and negative arrows in the instance language, it is possible to draw pictures that lead to the reasonable interpretation that a user has access to a file and to the reasonable interpretation that the user does not. The semantics of ambiguity is well-defined[MTW90], but is somewhat complex, so we do not reproduce it here. The Miro editor enforces well-formedness, but does not require that the pictures drawn are always unambiguous. For this reason, we cannot write the equation:

well_formed(ipic) = = all_arrows_attached(ipic) \& rambiguous(ipic).

Finally, most of the editor's procedures work on pictures regardless of whether they are drawn in the instance or constraint language. For example, moving a collection of boxes behaves the same regardless of whether they are instance or constraint boxes. To avoid duplicating the entire interface (e.g., have two separate Move Instance Picture and Move_Constraint_Picture procedures) we introduce a union sort $P$ to handle both instance and constraint pictures - just as we introduced an Object sort to handle both boxes and arrows. Figure 9 shows the signature for the PicUnion trait. By providing a union sort $P$ and the appropriate operators, the editor's procedures can now work on either type of Miro picture. Most of the operators introduced in PicUnion trait deal with coercing to and from values of the picture sort $P$ and values of instance and constraint picture sorts. 


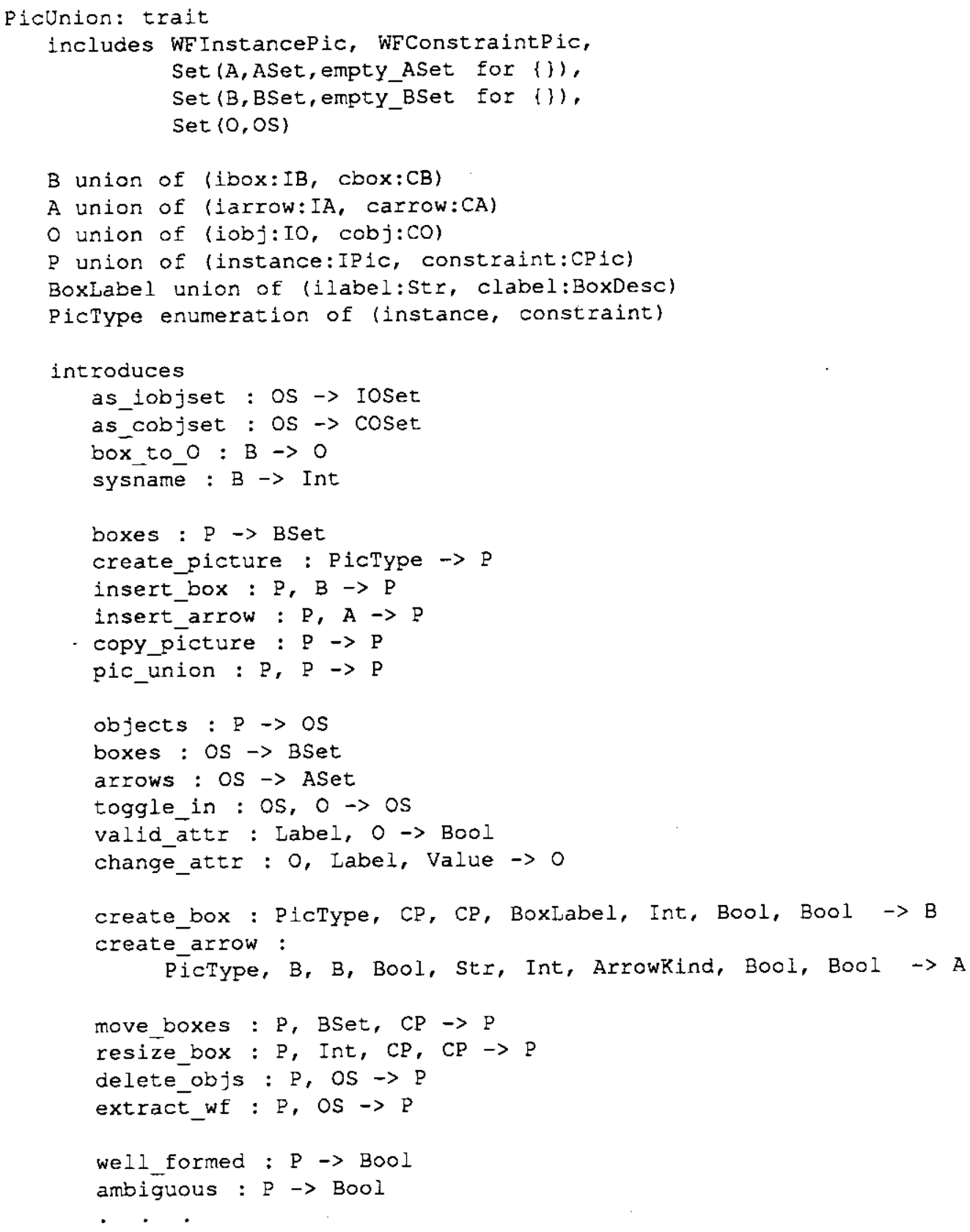

Figure 9: Part of the PicUnion Trait 


\subsection{Editor}

Now that we have a way of describing Miro pictures, we are ready to describe the editor's effects of manipulating them. We first describe the editor state at the trait level and then use interfaces to describe state changes. We ignore much of the lower-level detail (e.g., mapping to mouse and keyboard actions, how text interaction works); these are described informally in the Editor User's Guide[Zar90].

\subsubsection{LSL Level}

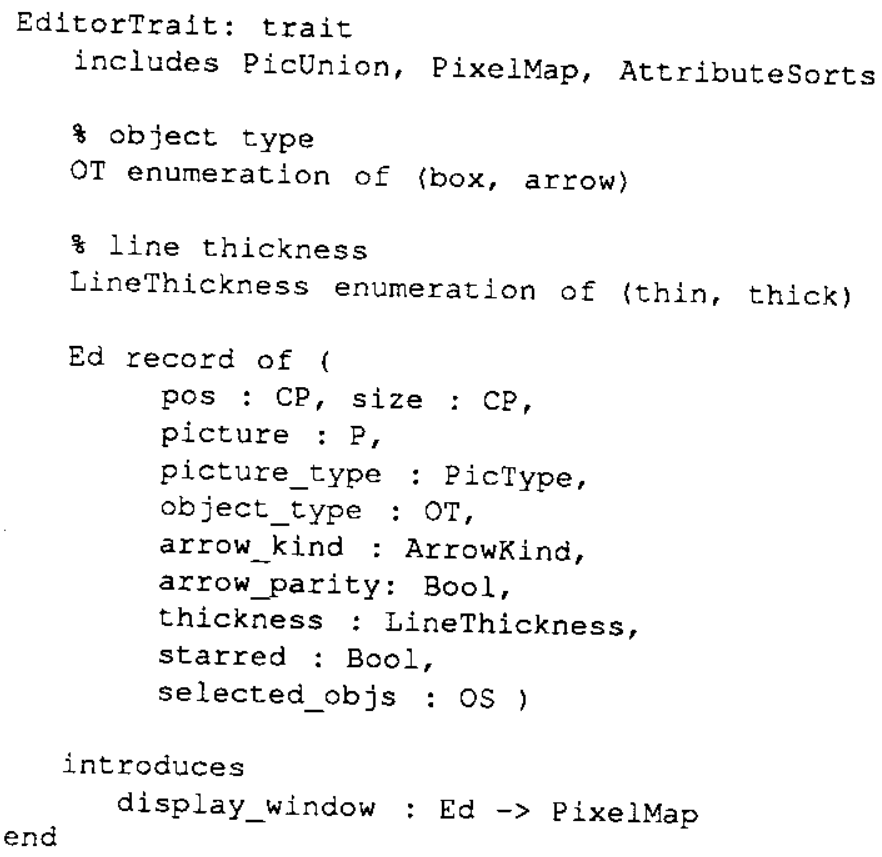

Figure 10: The Editor Trait

The Editor trait (Figure 10) introduces the sort, $E d$, which we use to model the editor state, again via the record construct. The pos and si ze fields indicate the location and size of the editor window on the screen. The picture field contains the current Miró picture, of sort $P$ (introduced in the PicUnion trait). Selected_objs is the set of currently selected objects in the picture. The remainder of the record describes the current "mode" of the editor (as indicated in the menus): picture_type indicates whether the current picture is an instance or constraint picture, object_type is either box or arrow, arfow_kind is the kind of arrow - syntactic, semantic or containment (relevant for only constraint pictures), and the rest of the attributes are self-explanatory. EditorTrait introduces one additional operator: display_window is left unspecified here, but is intended to be a mapping from the abstract editor value to actual screen pixels.

\subsubsection{Interface Level}

Now that we have build up a rich trait, we are ready to specify the editor's interface. First we name the module we are specifying (mi ro_editor), and then establish correspondences between types and sorts. The invariant specifies properties that must be true after every procedure and before all procedures except those named in the initialized by clause. Here, the invariant states that the picture maintained by the editor is always well-formed. 


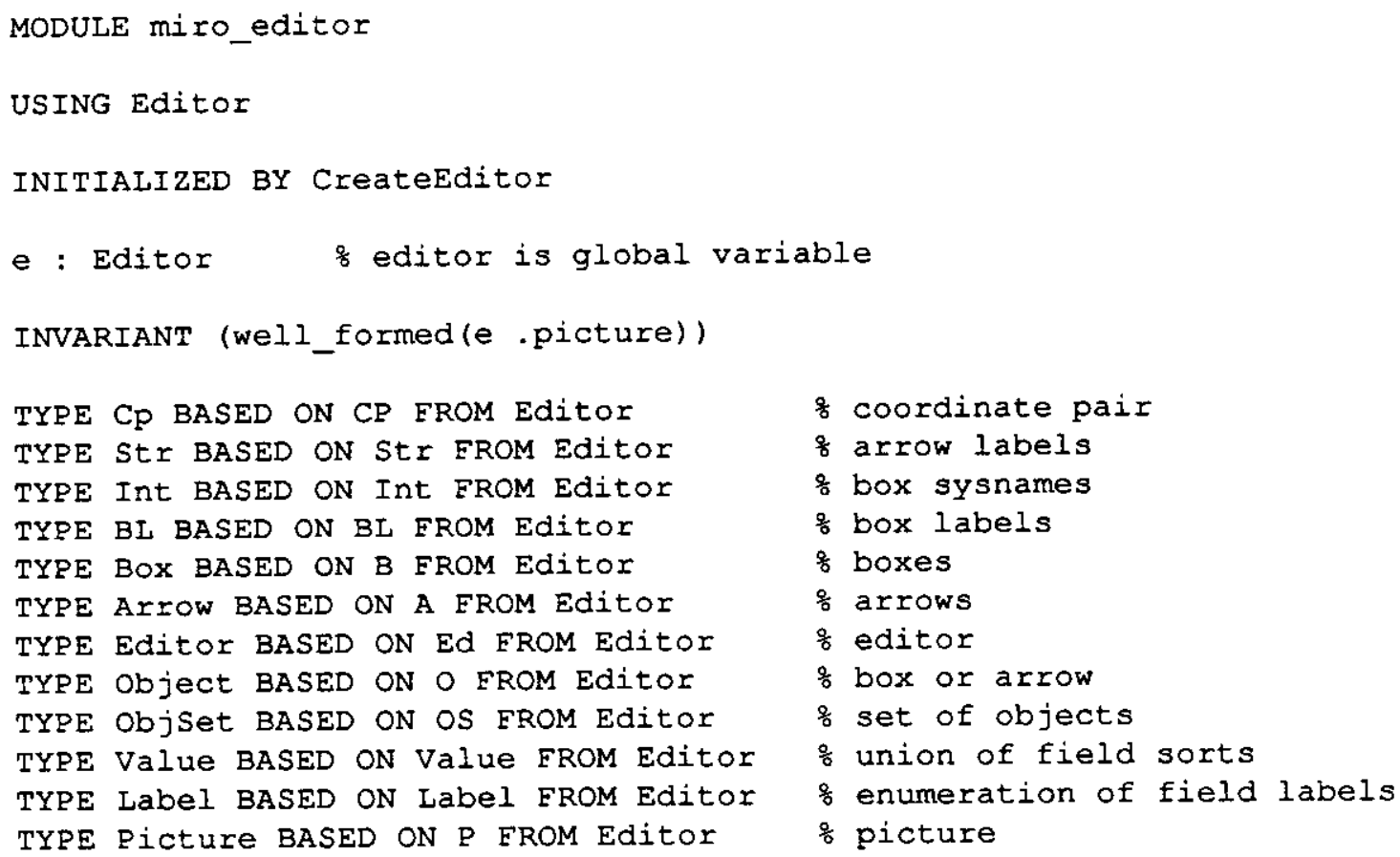

There are twelve procedures specified in the full specification. They include creating an editor, drawing a box, drawing an arrow, selecting an object, selecting a group of objects, unselecting objects, moving boxes, resizing boxes, deleting objects, copying objects, changing an object's attribute, and clearing the editor. Below we walk through only three to show what information is typically captured in an interface and how we rely on the traits to make this precise.

CreateEditor is the procedure that gets things started. Its effect is to initialize a new editor object with the default initial modes:

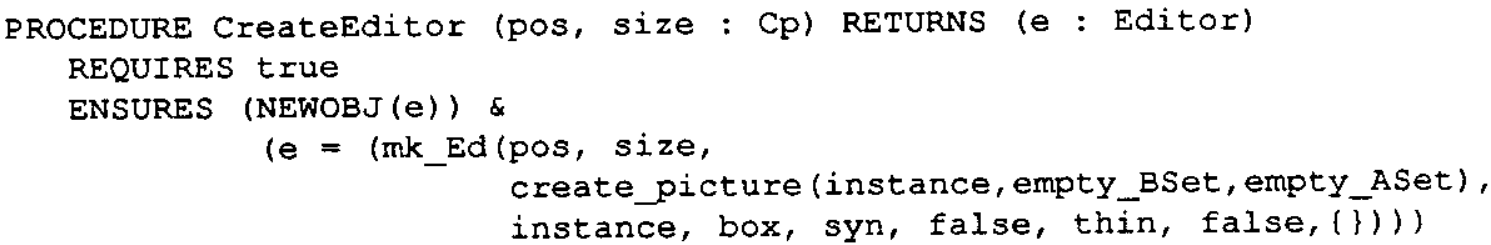

Resizing in the editor is fairly restrictive: a user can resize only boxes (not arrows), and must resize exactly one at a time. The third conjunct of the requires clause captures these restrictions. The hard "work" in the the ensures clause is done by a call to the trait operator resi ze_box defined for pictures; additionally the post-condition states that once a resize operation is performed all objects are unselected.

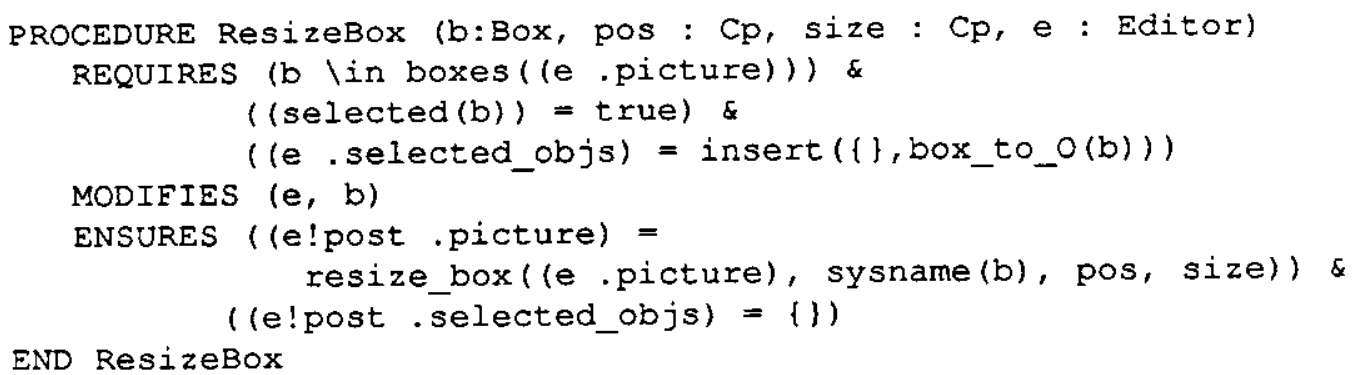


The copy operation in the editor is somewhat complex because of the well-formedness constraint. Copy operates on a subset of the currently selected objects, namely the maximal well-formed subset (i.e., all objects except "dangling" arrows). The ensures clause of Copyobjs thus says the new picture object, newpic, is the result of copying the well-formed subset of the selected objects of e.picture. e!post.picture is then the result of combining the existing picture with newpic, which has been moved by delta. CopyObjs also unselects all objects.

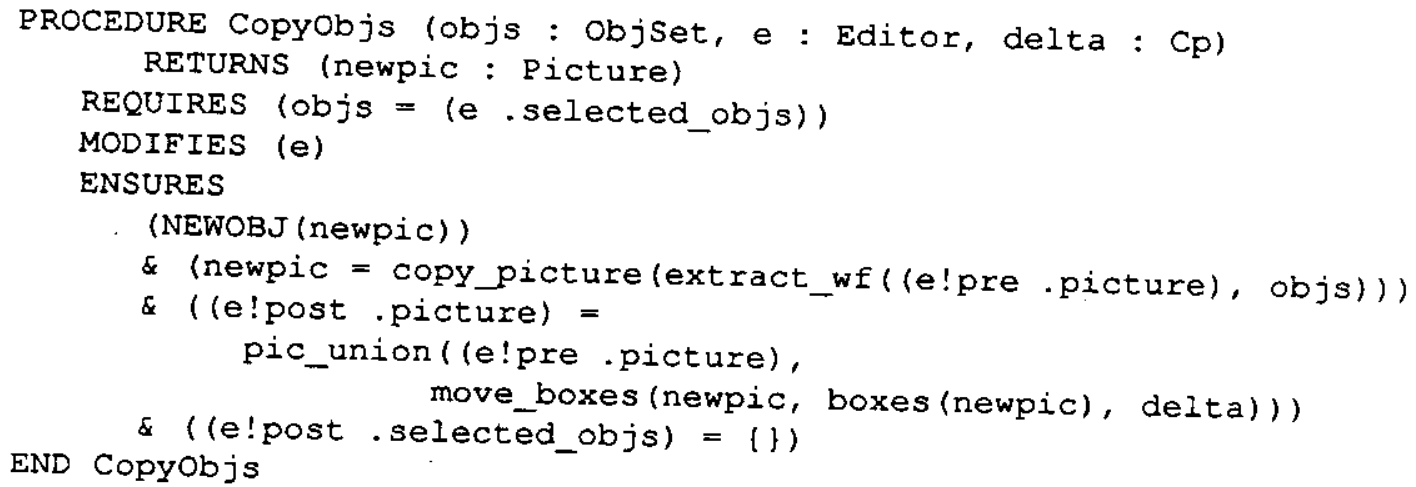

\section{Discussion}

We first discuss some properties about Miró pictures and the editor that are provable consequences of our specification, then two limitations of Larch that arose out of this specification exercise, and finally some general lessons learned from having done the specification after the implementation.

\subsection{Stating Consequences}

Larch provides a way to state consequences of a trait's theory through an implies clause. This clause is a good place to document additional assertions about a specificand. As a simple example, and also one that shows the interplay subset of selected objects that form a copying objects. At the interface level the CopyObjs procedure copies only the BasicPicture trait to copy only the well-formed picture. We could have defined the copy-picture operator in the at the interface level, leaving the trait level med subset but decided it was more appropriate to specify this restriction picture. We add the following implies clause to general: the copy-pict ure operator copies all the objects in the that we cannot make the stronger statement that the BasicPicture trait in order to record this decision explicitly. Note all of the fields, e.g., box labels, are copied.

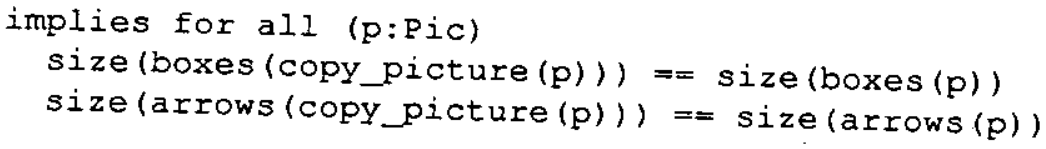

We can also state the strong assertion that a well-formed picture is actually just a graph where boxes are nodes and arrows are edges. Thus, we add the following consequence to the WFPicture trait:

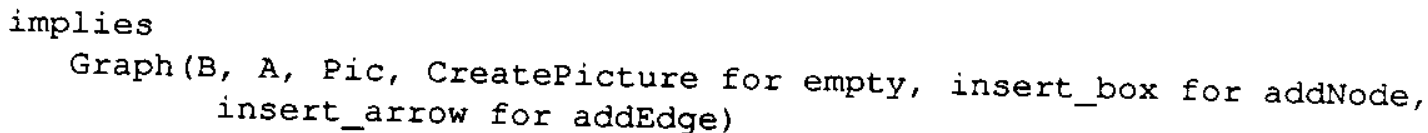


Finally, we can take this one step further. If we add to the WFInstancePic trait the requirement that each arrow goes from a user box to a file box and no arrow goes from a box of one type to a box of the same type, we can state as a consequence that a well-formed instance picture is a bipartite graph.

\subsection{Assessment of Larch}

\subsubsection{Subtyping Records}

We made a critical design decision by representing each Miró graphical object as a record. Records conveniently let us associate attributes with each kind of object and give us operators that let us set and get values of each of those attributes. However, the main drawback to using records is that Larch does not permit record "subtyping". It would be more general to define a GraphicalObject trait that introduces a record sort GO with fields like 1 abe 1 and then to define Box and Arrow traits, introducing B and A sorts, each as a "subtype" of GO. B would "automatically" have the same fields as GO plus ones like position and size; A would add fields like from_box and to box. Then we could write for $b$ of sort $\mathrm{B}, b .1$ abel and $b$. size, and for $a$ of sort A, $a .1$ abel and $a$. frombox. However, in Larch, if we were to factor out attributes common to all Miro objects into a GraphicalObject trait, we would be forced to use nested records in the records in the Box and Arrow traits and to write b.go. label or $a$. go.label where go is the field name of sort GO.

Instead, we decided to avoid nesting records entirely since the resulting specifications would be less readable. However, this decision forced us to include attributes of records in some traits that make sense only for subsequent uses of that trait. For example, the Box trait's record has a thick attribute that makes sense only for constraint, not instance, pictures. This kind of problem and solution is well-known in the "object-oriented" community; not until writing this specification did we see that record subtyping would not only be convenient, but lead to better specifications.

\subsubsection{Union Sorts}

Sort checking is invaluable in Larch, but one place where it gets in the way is in the use of unions. In the PicUnion trait, the $P$ sort is introduced to be the union of instance and constraint pictures. In the editor, it does not matter we have an instance picture or a constraint picture, we just want to select an object or copy objects. So, if the operators copy-picture: P $\rightarrow>$ P, copy picture: IPic $->$ IPic and copy-picture: CPic $->$ CPic are defined, then ideally we would like the constraints on copy-picture to hold regardless of whether the picture is an instance or constraint. Instead we are forced to first determine whether the picture is an instance (or a constraint) picture and then coerce the picture to be an instance (or constraint) picture so that we can apply the appropriate, more specific, copy operator, and finally, coerce back the result into the more general picture sort, $P$ :

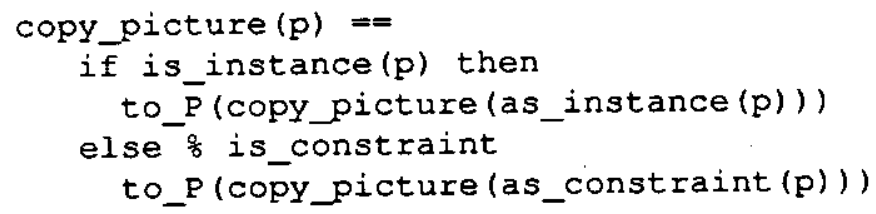

Another variation of this problem arose from specifying the change att $r$ operator in the Picture trait. We would like to specify change_att $r$ with the following simple equation:

$$
\text { change_attr(obj, label, value) }==1 \text { abel_gets (obj, value) }
$$

but we cannot for two reasons. First, an LSL operator name is an unstructured identifier - label_get s really stands for a set of possible identifiers depending on what the actual name of 1 abel is in the left-hand side of the equation 
above. Instead, we are forced to use a large if-then-else statement to cover each possible label. Second, the obj and value parameters to change_att $r$ are union sorts. As argued above, we need to do explicit coercion between the union sort and the specific sorts of the union (and vice versa) in order to achieve the intended effects of the above equation. Hence, change_att $r$ becomes one big two-layered if-then-else clause, first on object sort (box or arrow), then on label name. For each valid object/habel pair, there is a clause to do the appropriate coercions and assign the
value to the appropriate label:

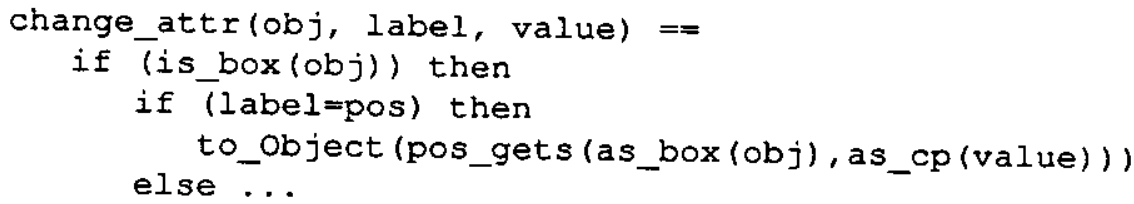

\subsection{Implications of Specifying After Implementing}

We began writing formal specifications in parallel with designing the Miro languages and designing and implementing of the editor. We wrote three major versions where the last version (this one) was written after the implmentation was running. The current version itself went through at least six minor iterations. Writing a formal specification after an implementation has two obvious implications. One is that the specification tends to be biased towards the implementation; the other is that places for improving the implementation become embarrassingly evident. We found both to be true in our case.

Having already implemented the editor before completing the specification, we had a concrete model of the languages and editor in mind. This model led to an implementation-biased initial specification. In each subsequent iteration we removed some of the "implementation details." We believe the final specification is relatively unbiased, implementation.

One example of implementation detail we removed is in the definition of well-formedness. In the implementation of the editor, the condition that all arrows be attached to boxes is enforced automatically by checking and reestablishing certain constraints on arrow objects. So, in a previous version of the specification, the arrow sort $A$ had fields for the coordinate positions of the arrow's head and tail. The WFPicture trait defined an operator called adjust_arrows that took a picture and a box, and based on the position of the boxes at the arrow's head and tail, algorithmically recomputed the new coordinates. But at a more abstract level, coordinate information for an arrow is not important; we only need to know which boxes are at its head and tail. As a result, we define an abstract well-formedness property (e.g., all arrows are attached) at the trait level and require through an interface invariant that all editor procedures enforce this property. No where in the current specification do we ever give a precise algorithm for enforcing well-formedness. It would be up to the implementor to decide whether and how arrows need to be adjusted to maintain well-formedness.

The second observation from doing the specification after the implementation is that doing the specification earlier would have resulted in a better implementation. One example to support this argument is our experience in implementing multiple selection in the editor. The initial implementation of the editor allowed at most one object to be selected at any time. While working on the specification we decided to extend the editor to allow selecting multiple objects. So before coding multiple selection, we wrote a mostly formal specification defining precisely what multiple selection that was clean, consistent be on the set of selected objects. The result was an implementation of multiple selection that was clean, consistent and relatively easy to add to the editor. 


\section{Final Remarks}

\subsection{Related Work}

The largest collection of Larch traits is the Larch Handbook[GHW85] (990 lines). The extended example in [GGH90] specifies some traits for a simple windowing system. Larch has also been used to specify properties of objects in a transaction-based distributed system[Win88]. To our knowledge, our specification is the largest Larch specification ever written and the only written for a "real" system.

In our specification, we assume that details about how Miro pictures are represented on the screen and what keyboard and mouse inputs activate the specified procedures are specified elsewhere. This in itself is a difficult problem, but has been addressed by others ([GGH90] and[Bow89]). Thus, we chose to focus at the next level of detail: properties of pictures and the editor. We have not seen any other specifications that deal with an interactive window application.

\subsection{Further Work}

As with any specification (or program), ours can be improved. We can generalize our traits to be suitable for more general graphical editors. We can extend the traits and interfaces to describe the more intricate behavioral aspects of the editor (e.g., other menu operations). However, the more interesting and challenging work we would rather pursue is to do mechanized proofs given that we have a formal specification and the Larch Prover [GG89]. There are two kinds of proofs we could perform: showing additional properties hold (e.g., the consequences discussed in Section 4.1 or the well-formedness invariant), and showing that the implementation of the editor satisfies our specification. There is evidence [GGH90] that the Larch Prover could "easily" be used to do the first kind of proof; doing the second kind would entail extensions to the Larch Prover itself.

\section{Acknowledgments}

We thank Allan Heydon, Rick Lemer, Mark Maimone and Doug Tygar for their extremely helpful comments on earlier drafts. We are also indebted to Brad Myers and the rest of the Garnet group for help in implementing the Miro editor.

\section{References}

[Bow89] Jonathan Bowen. Formal specification of window systerns. Technical Report PRG-74, Oxford University Computing Laboratory, June 1989.

[Che89] Jolly Chen. The Larch/Generic Interface Language. S.B. Thesis, MIT, May 1989.

[GG89] S.J. Garland and J.V. Guttag. An overview of LP, the Larch Prover. In Proceedings of the Third International Conference on Rewriting Techniques and Applications, pages 137-151, Chapel Hill, NC, April 1989. Lecture Notes in Computer Science 355.

[GGH90] Stephen J. Garland, John V. Guttag, and James J. Horning. Debugging Larch Shared Language specifications. Technical report, DEC-SRC, 1990.

[GHM90] J.V. Guttag, J.J. Horning, and A. Modet. Report on the Larch Shared Language: Version 2.3. Technical report, DEC-SRC, 1990.

[GHW85] J.V. Guttag, J.J. Horning, and J.M. Wing. Larch in five easy pieces. Technical report, DEC-SRC, 1985. 
[HMT ${ }^{+90]}$ Allan Heydon, Mark W. Maimone, J.D. Tygar, Jeannette M. Wing, and Amy Moormann Zaremski. Mir6: Visual specification of security. IEEE Transactions on Software Engineering, 16(10):1185-1197, October 1990.

[MTW90] Mark W. Maimone, J. D. Tygar, and Jeannette M. Wing. Formal semantics for visual specification of security. In S.K. Chang, editor, Visual Languages and Visual Programming. Plenum Publishing Corporation, 1990. A preliminary version of this paper appeared in Proceedings of the 1988 IEEE Workshop on Visual Languages, October, 1988, pages 45-51.

[Win88] Jeannette M. Wing. Specifying Avalon objects in Larch. Technical Report CMU-CS-88-208,CMU, 1988.

[Zar90] Amy Moormann Zaremski. The Miro editor: A user's guide. Miro Note 7, Carnegie Mellon University, School of Computer Science, 1990. 